\title{
Fundholding in Northern region: the first year
}

\author{
John Newton, Michelle Fraser, John Robinson, Dave Wainwright
}

\begin{abstract}
Objective-To describe the experiences of 10 fundholding practices in the Northern region during 1991-2 and to elicit subjective assessments of the impact of their change in status on practice management and patient care.

Design-Semistructured interviews were conducted with clinicians and practice managers; other staff in the practices were asked to fill in questionnaires. Questions were asked about the preparatory year, the impact of fundholding on clinical practice and practice management, perceptions of the costs and benefits of fundholding, and views about the
\end{abstract} future of the scheme.

Setting-10 of the 28 first wave fundholding practices in the Northern region, March-July 1992.

Results-Two interviews were conducted in nine practices and one interview in the tenth practice. Replies to the questionnaire were received from 35 general practitioners $(73 \%)$ and $89(58 \%)$ nonmedical staff. Practices sought independence in applying for fundholding status and found the preparatory year challenging and time consuming. General practitioners thought that the greatest change had occurred in relationships with consultants and the least change in relationships with patients. Most respondents thought that fundholding had changed the way they worked. The perceived benefits of fundholding were mentioned more often than the perceived costs.

Conclusions-The results offer some encouragement to the proponents of fundholding, but more longitudinal studies are needed to evaluate the misgivings of critics of the scheme. Fundholders are uncertain about their ability to make savings year after year, particularly in an increasingly cost contained environment.

\section{Introduction}

The introduction of fundholding in April 1991 was accompanied by a good deal of speculation about its impact on general practice. Some of this speculation was contained within general commentaries on the NHS reforms as a whole, ${ }^{1-3}$ and other commentators focused their attention on fundholding alone..$^{46}$ Opinion was divided on the potential costs and benefits of the scheme.

Those in favour of fundholding saw it as an opportunity for general practitioners to have a direct impact on access to and quality of secondary care, which they could now purchase on behalf of their patients. According to this view, significant benefits would accrue to both fundholders and patients. Fundholding practitioners would be less constrained in their clinical decision making and patients could anticipate more choice and improvements in services.

Opponents of fundholding expressed a range of concerns about the introduction of market principles into health care. Some thought that fundholding general practitioners had been given a perverse incentive not to spend money on their patients or even to be selective in the types of patient they enrolled on their list. ${ }^{3}$ Behaviour motivated by such economic criteria, it was argued, could only harm the doctor-patient relationship. ${ }^{5}$ It was feared that a scheme which allowed some practices and patients to be "winners" would necessarily result in other practices and patients being "losers." Later, these fears surfaced in a more public debate about the emergence of a two tier service.

The debate has been conducted on the basis of very little evidence. The government implemented its reforms without commissioning any evaluative or pilot studies and the experience of similar developments in the American health care system provided only limited comparisons. ${ }^{7}$ To date only two research studies, based on fundholding practices in the south of England, have published any results. ${ }^{89}$

The study reported here aimed to describe the experiences of 10 fundholding practices in the Northern region during the first year of the scheme $(1991-2)$. We were particularly interested in the practices' subjective assessments of the impact of this change on practice management and patient care.

\section{Methods}

A sample of nine practices was randomly selected from a list of fundholders stratified by the nine family health services authority areas in the Northern region. In one case two practices had joined the scheme as joint budget holders, making a total of 10 in the sample.

\section{DATA COLLECTION}

Three forms of data collection were used. They were piloted in another fundholding practice.

Firstly, two semistructured interviews were held at each practice with the lead clinician involved in fundholding and the practice manager between February and July 1992. Each audiotaped session lasted between one to one and a half hours. The first interview asked about becoming a fundholder; budget and contractual arrangements for 1991-2; impact on clinical practice; and impact on practice organisation and management. The second interview asked about the situation at year end 1991-2; the budget for 1992-3; contracts for 1992-3; management and organisational changes; patient services; information handling; and fundholding in the next three to four years.

Secondly, questionnaires were developed for self completion by other clinicians and non-medical staff in the practice. These aimed to elicit individual views about fundholding and details of the impact that it had had on their roles. Clinicians were asked about perceived costs and benefits of fundholding; involvement in fundholding; the impact of fundholding on work and on relationships with consultants and others; use of information technology; and feedback from patients. Non-medical staff were asked about their involvement in the decision to apply for fundholding 
status; perceived costs and benefits; and the impact of fundholding on work, use of computers, and patients. The questionnaires were designed so that responses would be given in one of three ways: a yes-no answer; a comment; or a choice from either three or five possible responses.

Thirdly, a schedule was distributed to practice managers, asking for information about the practice. This information was used to construct a profile of each practice.

\section{DATA ANALYSIS}

The audiotaped interviews were transcribed with a word processing package. Alongside this a freeform database enabled the creation of a set of topic headings under which summarised answers from each transcript could be listed. Within each topic heading the listed answers were then further coded to determine groupings of like answers. This computer assisted process of summarising and coding made a large amount of semistructured material amenable to qualitative analysis. ${ }^{10}$

Information from the structured questionnaire was entered on to computer spreadsheets. Simple statistical analysis of the spreadsheets resulted in totals and percentages of responses to each question for each practice. Comments and responses to the practice profile questionnaire were analysed with a more structured database program.

\section{Results}

In each of the 10 practices selected for the study the practice manager or lead fundholding clinician, or both, agreed to be interviewed. In nine practices two interviews were conducted: the first, in March or April 1992 , concentrated on a review of progress to date; the second, in June or July 1992, concentrated on plans for the coming year. In the same nine practices general practitioners and non-medical staff agreed to complete questionnaires. Thirty five replies $(73 \%$ of those distributed) were received from general practitioners and 89 ( $58 \%$ of those distributed) from non-medical staff.

\section{PREPARATION FOR FUNDHOLDING}

The first question in each of the opening interviews was why the practice had decided to apply for fundholding status. The most common reply was that the initiative offered an opportunity to be independent. This was expressed in terms of freedom to do certain things (refer to hospitals of their choice; spend money on new staff and equipment) and freedom from externally determined policies and procedures (for example, contracts set by the district health authority).

TABLE I-Involvement of general practitioners $(n=35)$ in fundholding

\begin{tabular}{lrcrc}
\hline & \multicolumn{4}{c}{ Degree of involvement } \\
\cline { 2 - 5 } Aspect of involvement & High & Medium & Low & None \\
\hline Decision to apply & 22 & 7 & 3 & 3 \\
Setting budget for 1991/2 & 8 & 7 & 18 & 2 \\
Negotiating contracts for 1991/2 & 8 & 6 & 20 & 1 \\
Monitoring budget for 1991/2 & 9 & 5 & 19 & 2 \\
$\begin{array}{l}\text { Planning budget and contracts } \\
\text { for 1992/3 }\end{array}$ & 11 & 8 & 14 & 2 \\
\hline
\end{tabular}

TABLE II-General practitioners' assessment of changes in relationships as a result of fundholding. Values are numbers $(n=35)$ replying to the question, "To what extent have your relationships with any of the following changed as a result of acquiring fundholding status?"

\begin{tabular}{lccccc}
\hline & $\begin{array}{c}\text { "A great } \\
\text { deal" }\end{array}$ & $\begin{array}{c}\text { "Quite a } \\
\text { lot" }\end{array}$ & $\begin{array}{c}\text { "To some } \\
\text { extent" }\end{array}$ & "A little" & "Not at all" \\
\hline Consultants & 5 & 8 & 9 & 6 & 7 \\
Patients & & 1 & 3 & 5 & 26 \\
Doctors in the practice & 1 & 4 & 5 & 8 & 17 \\
Other general practitioners & 1 & 4 & 7 & 6 & 17 \\
Practice support staff & 3 & 8 & 11 & 6 & 9 \\
Private sector health care organisations & 5 & 1 & 7 & 19 \\
\hline
\end{tabular}

For some, the scheme matched their self perception as being "progressive" and "innovatory" practices.

There was a high level of involvement in the decision to apply for fundholding status (table I). But involvement of general practitioners (other than the lead clinician) in other aspects of fundholding (such as setting and monitoring contracts) was at a lower level. There are indications, however, that their level of involvement was greater in year $2(1992-3)$ than year 1 (1991-2).

The day to day business of preparing for fundholding had been the task of the practice or fund manager(s) together with the lead clinician. Five of the nine practices had been collecting the types of data required by the regional health authority for several years, and all of the practices had computer systems in operation before the preparation period. Even so, most of the practices said that the data collection and processes of budget and contract setting were extremely demanding. This was attributed mainly to the need to liaise with a large number of external agencies and bodies either by telephone or in face to face meetings.

IMPACT OF FUNDHOLDING

Tables II and III show general practitioners' and non-medical staff's assessment of the impact of fundholding on their relationships and roles. Table IV shows how the practices ended the first year in financial terms, and Table $\mathrm{V}$ shows how general practitioners and non-medical staff assessed the costs and benefits of fundholding in their practices.

TABLE III-Views of general practitioners and non-medical staff on whether their jobs had been affected by the change to fundholding status. Values are number (percentage) of respondents

\begin{tabular}{lcc}
\hline & $\begin{array}{c}\text { "Has your job been affected by } \\
\text { the change to fundholding } \\
\text { status?" }\end{array}$ \\
\cline { 2 - 3 } & $\begin{array}{c}\text { Job had been } \\
\text { affected }\end{array}$ & $\begin{array}{c}\text { Job had not } \\
\text { been affected }\end{array}$ \\
\hline $\begin{array}{l}\text { General practitioners: } \\
\text { "From a medical point of view" }\end{array}$ & $17(49)$ & $18(51)$ \\
"From an administrative point of view" & $24(69)$ & $11(31)$ \\
Non-medical staff & $53(60)$ & $36(40)$ \\
\hline
\end{tabular}

General practitioners were asked to assess the extent to which they thought relationships with a number of other persons and organisations had changed as a result of acquiring fundholding status. The greatest amount of change was thought to have occurred in relationships with consultants: $13(37 \%)$ said that this had changed "a great deal" or "quite a lot." In the interviews six practices cited changes for the better, which included overcoming difficulties in getting terminations; improving discharge summaries; discussions about a protocol for infertility referrals, and, in a different practice, for back referrals. Apart from these particular initiatives there was a general enthusiasm for the way in which fundholding had opened up communication with hospital colleagues. One doctor summed up the change: "We've actually seen them for once; they've been out here visiting just to make sure that we are aware of their potential services."

The least amount of change was thought to have occurred in relationships with patients: just one doctor thought that this had changed "quite a lot." In the interviews most of the lead clinicians said that day to day relationships with patients had changed very little. Some patients had voiced initial apprehension about their continuing to receive medication, but most were thought to be neutral about fundholding. When asked how they thought patients had responded to the practice becoming a fundholder, $25(71 \%)$ general practitioners replied "neutrally." Practices, however, had not been proactive in advertising their change in 


\begin{tabular}{|c|c|c|c|c|c|}
\hline $\begin{array}{l}\text { Practice } \\
\text { No }\end{array}$ & List size & Total budget $(£)$ & $\begin{array}{l}\text { Budget per } \\
\text { patient }(£) \\
\end{array}$ & $\begin{array}{c}\text { Saving } \\
\text { (overspend) }(\mathscr{C})\end{array}$ & $\begin{array}{c}\text { Outcome as } \% \text { of } \\
\text { total budget }\end{array}$ \\
\hline 1 & 10500 & 1774347 & 169.98 & $(40000)$ & $-2 \cdot 25$ \\
\hline 2 & 9712 & 1463598 & 150.70 & 46000 & $3 \cdot 14$ \\
\hline $\left.\begin{array}{c}3^{*} \\
4^{\star}\end{array}\right\}$ & 16835 & 2023100 & 120.17 & 21116 & 1.04 \\
\hline 5 & 10500 & 1270445 & 120.99 & $(16500)$ & $\begin{array}{r}-1.30 \\
3.76\end{array}$ \\
\hline 6 & 14000 & 1673600 & 119.54 & 63000 & 3.76 \\
\hline 7 & 12500 & 1555475 & 124.44 & 61000 & 3.92 \\
\hline 8 & 9900 & 1399999 & 141.41 & 47000 & $3 \cdot 36$ \\
\hline 9 & 10800 & 1511511 & 139.95 & $194392 \dagger$ & $12 \cdot 86$ \\
\hline 10 & 13900 & 1637448 & 117.80 & $(50516)$ & $-3 \cdot 09$ \\
\hline
\end{tabular}

*Treated as consortium by Northern Regional Health Authority.

†Owing to flawed dataset, budget allocation of this practice was inflated; proportion of underspend was returned to data on transactions twice because of the lack of integration of fundholding and clinical software systems. It is not surprising, therefore, that in the interviews several practice managers reported increased tension and stress among staff as a result of the changes.

Table IV shows that seven of the 10 practices made overall savings in 1991-2 and that three practices overspent their budgets. Most of the practices making savings had done so on their hospital budgets, and this was attributed to a combination of setting favourable contracts, making fewer referrals, and doing more minor surgery in house. At the time of the second interviews (June or July 1992) it was not clear whether the regional health authority would treat all of the savings as "genuine."

Only two of the 10 practices were overspent on their hospital budgets, and these two practices also had an overall overspend. They both accounted for this outcome in terms of the rigidity of the block contracts they had been recommended to make by the regional health authority. The third practice had overspent because it had taken over a smaller practice after the preparatory work on budget setting had been completed, and it had not been able to make an accurate estimate of prescribing costs for the 1300 patients involved.

To summarise the impact of fundholding, general practitioners and non-medical staff were asked an open ended question about the costs and benefits of the scheme in their practice. The results are listed in Table $\mathrm{V}$ in descending order of frequency with which the items were mentioned. Both general practitioners and non-medical staff mentioned benefits more often than costs. The main benefit for general practitioners was improved practice management and computer systems (10 mentions); for non-medical staff it was swifter referrals (11 mentions). All respondents saw increased time and effort in administration, or simply "extra work," as the main cost of fundholding.

\section{Discussion}

In this study attitudinal data were collected by means of interviews and questionnaires from 10 first wave fundholding practices in the Northern Regional Health Authority. We found that doctors and nonmedical staff thought that fundholding had altered their roles and relationships with each other and with agencies outside of the practices. Seven of the 10 practices had made an overall saving on their first year's budget, and everyone felt that the benefits of being in the scheme outweighed the costs.

These findings offer some encouragement to the proponents of the fundholding initiative. If fundholders were to be a lever for change in the reorganised National Health Service there is evidence from this study that they are achieving this objective. Each of the practices reported some improvement in the provision of hospital care or practice services that they felt could be attributed to the acquisition of fundholding status. All of the practices were looking forward to the second year in which the "steady state" of year 1 would give way to even greater freedom and opportunity.

Unfortunately these findings cannot support or reject the reservations held by critics of the scheme. The main misgivings relate to the perverse incentives fundholding was thought to create: not to refer patients; to shift cases from elective to emergency care; to select or deselect patients according to their cost liability. Such matters were occasionally raised in the interviews and always received vigorous denials that such considerations would ever influence decision making. Answers to these issues, however, require more longitudinal research designs and more rigorous forms of data collection than were possible in this

More of the non-medical staff said that fundholding had changed their job, and when asked to elaborate said that they had been involved in a lot of extra work. One example of this was the frequent necessity to enter 
study. There is clearly a need for more evaluative studies in this area.

Although all of the practices were looking forward to continued participation in the scheme, there were some concerns about the future. A variety of difficulties were foreseen about the ability of fundholders to continue making savings. Some realized that their first year savings were intrinsically "once only": "We can't go generic every year," said one lead clinician. One doctor raised the issue of random variations in service needs addressed in a paper by Crump et al." As he put it: "You could blow your budget by $15 \%$ without consciously doing anything." For all these reasons the future was thought to be uncertain. Some practices were therefore thinking again about employing additional professionals out of budget surpluses. Such arrangements, it was argued, required a more reliable source of income than they could currently envisage, and a clearer indication from the region (or the Department of Health) concerning policy about the retention and spending of savings.

A related concern stemmed from predictions about the direction of health care funding. Given the demands of the United Kingdom's aging population and the tendency of health care costs to rise, it seemed certain to some doctors that governments' policies in this area would contain an appreciable element of cost containment. A primary health care sector with half or more of the general practitioners as fully fledged budget managers would certainly facilitate the implementation of such a strategy, as has been pointed out. ${ }^{12}{ }^{13}$ It would then be a classic example of double talk if an initiative that was sold to general practitioners as a vehicle for enhancing freedom turned out to be a means of constraining it.

We thank the Research Initiatives Fund at the University of Northumbria at Newcastle for its financial support and doctors and staff in the 10 selected practices for giving us their time. Thanks are also due to Dr Colin Leon, consultant adviser in primary health care at the Northern Regional Health Authority, for introducing us to the practices and Dr Allen Hutchinson, programme director at the Centre for Health Services Research at Newcastle University, for commenting on an earlier draft of this paper.

1 Bevan G, Holland W, Mays N. Working for which patients and at what cost? Lancet 1989;i:947-9.

2 Brazier JE, Normand CEM. An economic review of the NHS white paper. Scottish Fournal of Political Economy 1991;38:96-105.

3 Mullen PM. Which internal market? The NHS white paper and internal markets. Financial Accountability and Management 1990;6:33-50.

4 Forbes JT. Practice budgets-lifting the veil of ignorance. $f R$ Coll Gen Pract 1989;39:355-86.

5 Drummond $M$, Hawkes $R$, Marchment $M$. General practice fundholding.

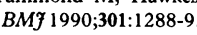

6 Roland $M$. Fundholding and cash limits in primary care: blight or blessing? BMF 1991;303:171-2.

7 Weiner J, Ferris P. GP budget holding in the UK: lessons from America London: King's Fund Institute, 1990. (Research report No 7.)

8 Glennerster H, Matsaganis M, Owens P. A foothold for fundholding. London: King's Fund Institute, 1992. (Research report No 12.)

9 Glynn J, Murphy MP, Perkins DA. GP practice budgets: an evaluation of the financial risks and rewards. Financial Accountability and Management financial risks

10 Berg BL. Qualitative research methods. Needham Heights, MA: Allyn and Bacon, 1989 .

11 Crump BJ, Cubbon JE, Drummond MF, Hawkes R, Marchment MD Fundholding in general practice and financial risk. BMF 1991;302:1582-4. 12 Dingwall R, Hughes D. Joe Stalin and the NHS revolution. Health Service foumal 1991;101:23-4.

13 Melzer D. Supermarket fantasies. Health Service Journal 1992;102:17

(Accepted 9 December 1992)

\section{Lincolnshire agricultural workers' disease}

All my life I was research minded but had no training in the discipline. When I started in practice in Lincolnshire I found that agricultural workers, blacksmiths, and saddlers seemed unduly prone to respiratory disease and disablement. Professor Pepys at the Brompton Hospital elucidated the diseases of blacksmiths and saddlers, whose numbers were gradually falling, but the disease of the agricultural workers took up more and more of my attention.

In the mid-1930s I studied the work of the farms, which then included stacking corn crops in the fields to ripen and dry (if possible). The sheaves were stacked in a certain way in stooks, each of about 12 sheaves. The corn was left to ripen until the farmer judged it was fit, then it was loaded on wagons, brought to the stackyard, and made into stacks. All these operations were highly skilled, and a well filled stackyard in late autumn was a beautiful sight. Though none of these jobs seemed dangerous, the stack holding as it did the ripe grain safely, also contained many moulds and much other dust.

On threshing days the thresher came with his crew. His equipment was a steam traction engine, used to drive the machine by belts. The main machine was the thresher itself, which (the sheaves having been opened and the stems separated) shook the corn and banged it against a metal grille. The grain fell through to be collected, the straw was collected and stacked-and the chaff? That mostly blew away-threshing days always seemed windy -but someone collected as much as possible in sacks. The engine put forth a lot of black smoke at a low level. And everyone, especially those downwind, had to breathe dust and smoke.

As time went by I found that men fell ill with respiratory disease after threshing. Some had severe asthma, which did not respond to any remedy available then. At least one died. A young owner of a threshing set had to sell his machines and retire from the job. Some had chronic asthma, which went on for months, so that the patient had to leave farm work and get work in the city. Mostly these men never had asthma again and lived out normal lives. But apart from those with asthma, many who had served long on farms had a vague illness with breathlessness and some wheezing, which took weeks to resolve. Rales were frequently to be heard. $x$ Rays were reported clear. If the man continued at work threshing he would become permanently disabled.

This complaint, which does not seem to have been observed or described before, put me in a quandary. I knew that the man should receive what was then known as "workmen's compensation," but for this to be paid a consultant had to confirm my opinion. The disease looked so definite and unique to me, but nobody else could or would recognise it. I lived in a state of rage for years. My cases were returned by the local consultants with the advice that "As far as I am concerned this is a case of chronic bronchitis."

Of course the National Union of Agricultural Workers was interested and eventually the general secretary arranged for a patient to see Dr Chris Darke of Sheffield Royal Infirmary. Dr Darke came down to rural Lincolnshire with technicians and investigated the patients. He confirmed my opinion that Lincolnshire farmers' disease was an occupational disease, probably caused by allergy to mould spores in the dust. This was in the late $1960 \mathrm{~s}$ and the latest farming revolution was well under way. Cereals were no longer reaped or stacked but harvested by giant combine harvesters and the grain stored in corn driers. But Dr Darke was able to pinpoint new dangers: in particular, the harvesters threw up dense clouds of dust, as did the air blown through the corn driers by huge fans. So the men were still at risk.

His work was mainly published in an agricultural journal and was enormously influential. The farmers (who no doubt had had a touch of the disease themselves) at once saw the importance of the report. Almost overnight, harvesters sprouted dust free cabins for the driver, and in the corn driers precautions were taken. The disease disappeared. As I drive about the countryside at harvest time and see the combine harvesters with their dust free cabins, I feel a little proud.-W PATRICK ROE, retired general practitioner, Welwyn Garden City 